\title{
The enamel thickness of gestational diabetes mellitus rat offspring
}

\author{
Nurdiana Dewi, ${ }^{1}$ Ahmad Syaify, ${ }^{2 *}$ Ivan A. Wahyudi ${ }^{3}$
}

\section{Abstract}

Objective: Gestational Diabetes Mellitus is diagnosed when Diabetes Mellitus begins or is first detected during pregnancy. Enamel hypoplasia is an abnormality in tooth enamel that was common found in children born to diabetic mothers. The aim of this study was to determine the molar enamel thickness of rat offsprings born to diabetic rat.

Material and Methods: This research used sixteen rat offsprings born to 8 diabetic rats who had been injected with STZ dose of $40 \mathrm{mg} / \mathrm{kg}$ and 16 rat offsprings born to 8 control rats. Rat offsprings were decapitated on day 5 after birth and taken right mandibular molar tooth germ.
Tooth germ then embedded in paraffin block. Haematoxylin eosin staining performed for histological analysis. Enamel thickness measured on the images using ImageJ Software at 5 field of view. Results: The enamel thickness on the DM group $(333.02 \pm 135.81 \mu \mathrm{m})$ were lower than control group $(415.39 \pm 146.17 \mu \mathrm{m})$. Mann Whitney test results showed a significant difference between the DM and control group.

Conclusion: It was concluded that enamel thickness decrease in gestational diabetes mellitus rat offspring.

Keywords: Enamel thickness, Gestational Diabetes Mellitus, Tooth germ

Cite this Article: Dewi N, Syaify A, Wahyudi IA. 2021. The enamel thickness of gestational diabetes mellitus rat offspring. Journal of Dentomaxillofacial Science 6(3): 193-196. D0l: 10.15562/jdmfs.v6i3.1130

'Department of Pediatric Dentistry, Faculty of Dentistry, Universitas Lambung Mangkurat, Banjarmasin, Indonesia

${ }^{2}$ Department of Periodontology, Faculty of Dentistry, Universitas Gadjah Mada Yogyakarta, Indonesia ${ }^{3}$ Department of Biomedica, Faculty of Dentistry, Universitas Gadjah Mada Yogyakarta, Indonesia

*Correspondence to: Ahmad Syaify, Department of Periodontology, Faculty of Dentistry, Universitas Gadjah Mada Yogyakarta, Indonesia ahmad.syaify@ugm.ac.id

Received: 26 December 2020 Revised: 25 March 2021 Accepted: 27 July 2021 Available 0nline: 1 December 2021

\section{Introduction}

The formation of primary teeth or odontogenesis begin at the age of 6-7 weeks intrauterine. Growth and development of this teeth include initiation, proliferation, histodiferentiation, morphodiferentiation, apposition, calcification, maturation and eruption. ${ }^{1,2}$ Enamel formation or amelogenesis was enamel matrix formation process which followed by enamel calcification. Distractions that occur in enamel matrix formation can cause enamel hypoplasia, whereas disturbance at the time of calcification may lead to hypocalcification. ${ }^{3}$ Gestational Diabetes Mellitus is reported to cause enamel hypoplasia in children born. ${ }^{4}$

Diabetes Mellitus (DM) is a metabolic disorder characterized by hyperglycemia that occurs due to abnormalities of insulin secretion, insulin action, or both. ${ }^{5}$ Gestational Diabetes Mellitus is diabetes that first detected during pregnancy. ${ }^{6}$ Pregnancy is normally accompanied by progressive insulin resistance that begins near mid-pregnancy and progresses through the third trimester to levels that approximate the insulin resistance seen in individuals with type 2 diabetes. Insulin resistance is caused by combination of an increase in maternal adiposity and the insulin-desensitizing effects of placental hormones such as progesterone, cortisol, placental lactogen, prolactin and growth hormone. Pancreatic $\beta$ cells normally increase insulin secretion as compensation for insulin resistance during normal pregnancy. As a result, changes in circulating glucose levels over the course of pregnancy are quite small compared with the large changes in insulin sensitivity. Beta cell ability in resolve progressive insulin resistance is the hallmark of normal glucose regulation during pregnancy. Patient with Gestational DM can not provide enough insulin to get normal blood glucose regulation, resulting in elevated blood glucose levels.?

Subsequenly, disorders that occur during pregnancy can cause disturbances in the formation of teeth, one of which is enamel hypoplasia often found in the enamel of children born to mother with DM. Enamel hypoplasia is a condition of reduced enamel thickness due to interference of the secretory stage of amelogenesis. Defective enamel sites may provide suitable local environment for adhesion and colonization of cariogenic bacteria, and bacteria may retain at the base of the defect in contact with exposed dentin, thus dental caries on these defective sites may develop more rapidly. ${ }^{8}$ Enamel hypoplasia predisposes to the occurrence of Early Childhood Caries (ECC) and dental caries. ${ }^{9,10}$ Previous study showed enamel hypoplasia in Wistar rat offsprings born to alloxan-induced diabetes mellitus rats. ${ }^{4}$ This study aims to determine the thickness of molar tooth germ enamel of Wistar rat offsprings born to Gestational Diabetes Mellitus rats. 


\section{Material and Methods}

This research is an experimental research. Research procedure was approved by the Ethics and Advocacy Unit Faculty of Dentistry Universitas Gadjah Mada.

Treatment of experimental animals conducted at the Laboratory of Integrated Research and Testing Unit IV Universitas Gadjah Mada. The experimental procedure was approved by the Ethics and Advocacy Unit Faculty of Dentistry Universitas Gadjah Mada. Sixteen female Wistar rats aged 2-3 months, 150$200 \mathrm{~g}$ body weight were used in this study. The rat were adapted in the cage environment for 1 week, given the standard feed and drink ad libitum. The rats were kept on a 12 -h light-dark cycle at $22-24^{\circ} \mathrm{C}$. After 1 week, the rat caged with male rats to mated. Morning when discovered sperm in vaginal smears indicates rat mated and expressed as gestation day 0 . Group A consisted of 8 female Wistar rats treated with intraperitoneal injection of streptozotocin (Sigma, MO, USA) $40 \mathrm{mg} / \mathrm{kg} \mathrm{BW}$ in the 0th day of pregnancy. Group B consisted of 8 female Wistar rats injected with citrate buffer $(0.05 \mathrm{M}, \mathrm{pH} 4.5)$ in the 0th day of pregnancy. Subsequent monitoring was performed by body weight weighing, palpation of the abdomen, and measurements of blood glucose levels of rats in the 0th, 7th, 14th and 19th day of pregnancy. Rats were declared pregnant when there was a noticeable increase in body weight and enlargement in utero on palpation $10-14$ days after sperm found. ${ }^{9}$ Measurement of blood glucose levels were done with Accu-Check Active (Roche, Germany). Rats expressed DM when having blood glucose $>120 \mathrm{mg} / \mathrm{dL}$ and showed signs of DM (polyphagia, polyuria, polydipsia). Two offsprings were taken at random from each rat, so there were 32 offsprings. Offsprings were anesthetized with ether before decapitation. The offsprings then decapitated on day 5 after birth. Mandibular molar tooth germ were taken and fixed with $4 \%$ PBS formalin. After the tooth germ samples were fixed for 24 hours, it were decalcified using EDTA 10\% at $4^{\circ} \mathrm{C}$ for 14 days then embedded in paraffin and cut with $6 \mu \mathrm{m}$ thickness. Sliced tooth sample were deparaffinizsed with xylol 2 times each for $10 \mathrm{~min}$, absolute alcohol 2 times each for 5 minutes and serial alcohol $95 \%, 90 \%, 80 \%$, and $70 \%$ respectively for 1 minute. Staining with hematoxylin staining was done in a jar for 3-5 minutes followed by washing with distilled water 3 times each for 1 minute. Sliced tooth sample then immersed in acid staining alcohol for 30 seconds, washed at water flow for 3-5 minutes and rinsed with distilled water 1 times. Samples were inserted in a solution of $1 \%$ eosin for 1-2 minutes, then washed with distilled water 3 times and dehydrate. Samples were depressed with anada balsam and covered with a glass lid.
Figure 1 observations were performed on the images using a camera connected to a microscope. Examination was conducted on 5 field of view. Two field of view at tip of bulge, two field of view at mid between tip and base of bulge, and one field of view at base of bulge. Enamel thickness measurements carried out on the boundary between ameloblast and enamel to the boundary between the enamel and dentin. Measurements were made perpendicular to the boundary between enamel and dentin layers. The measurement results were averaged for each sample and analyzed by Mann Whitney test.

\section{Results}

The body weight and blood glucose levels of rat in both DM and control group were measured on days $0,7,14$, and 19 . The mean and standard deviation values of the measurement results can be seen in figure 2.

There was an increase in the body weight of rat on each day of observation both in the treatment and control groups. The result of Kruskall Wallis test even showed that there was a significant increase in the body weight of rats in both DM and control groups with a $\mathrm{p}$ value $0.00(\mathrm{p}<0.05)$. The results of palpation on the abdomen also showed an enlargement of the uterus indicating the presence of fetus.

Rat blood glucose concentration of DM group $>120 \mathrm{mg} / \mathrm{dL}$ at $7 \mathrm{th}, 14 \mathrm{th}$, and 19th day of pregnancy. Rat blood glucose concentration of control group $<120 \mathrm{mg} / \mathrm{dL}$. Mann Whitney test results showed $p=0.00(p<0.05)$ on day 7,14 and 19 which means there were significant differences in blood glucose levels of DM group and control group. ${ }^{11}$

In addition, the results of visual inspection on rats showed classic symptoms of DM. First, polydipsy (lots to drink) was known from the number of drinks (bottles) needed in the DM group higher than in the control group. Second, polyuria (lots of urine) was detected from rat droppings under the cage very wet. Third, polyphagia (much to eat) was known from the amount of food consumed by DM group higher than the control group. And asthenia (weak) was detected from the activity of the DM group tended to be more passive than the control group.

Hence, due to the increase in body weight, the enlargement of the abdomen, the increased blood glucose levels ( $>120 \mathrm{mg} / \mathrm{dL}$ ), as well as the classic symptoms of DM, the rats were declared to experience gestational DM. Besides, those rats were also declared to be pregnant due to the increase in body weight and the enlargement of the abdomen (uterus) 10-14 days after sperm was found in vaginal smears. ${ }^{12}$ Meanwhile, those rats were declared 


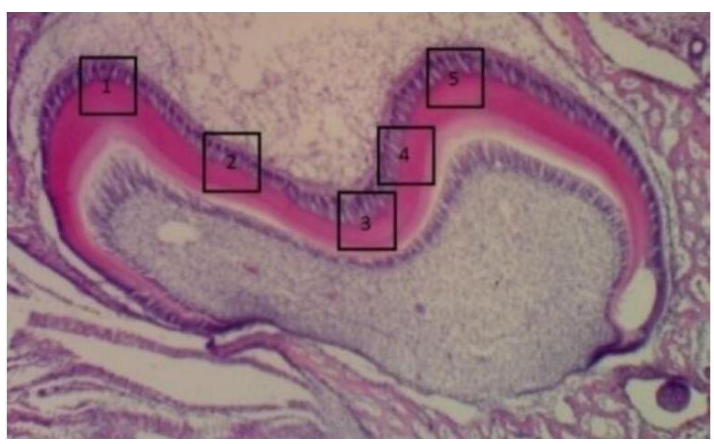

Figure 1 Field of view of histological observation

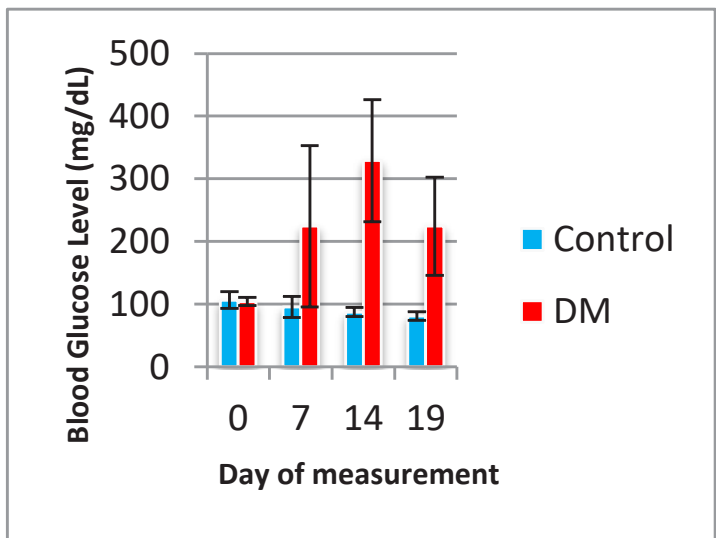

Figure 2 Rat body weight and blood glucose levels

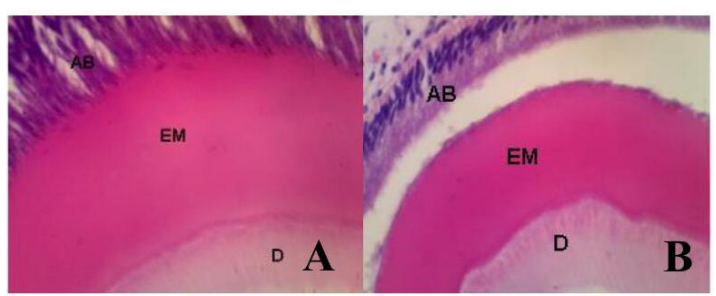

Figure 3 Microscopic cross-section of a molar tooth germ of rat at day 5 (observed at 400x magnification). A. Control group, B. Diabetes Mellitus group D Dentin; EM, Enamel; AB Ameloblasts

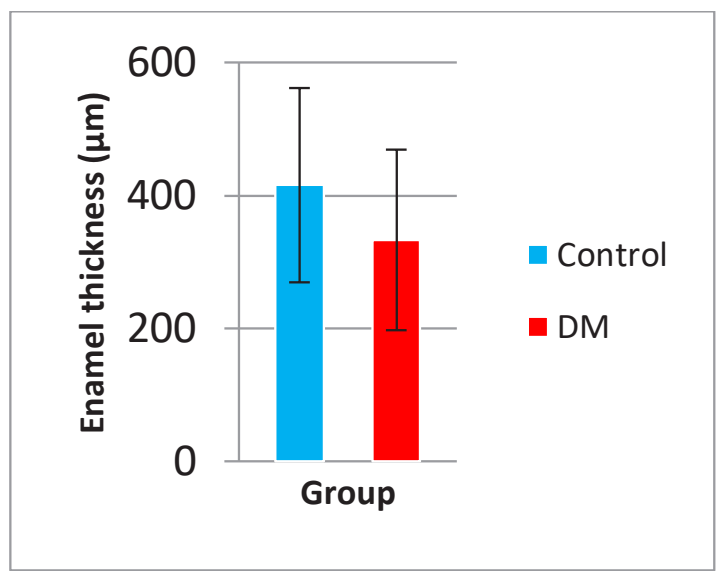

Figure 4 Mean and standard deviation of enamel thickness $(\mu \mathrm{m})$ to suffer DM since their blood glucose level was higher than $120 \mathrm{mg} / \mathrm{dL}$, and they also got classic symptoms of DM. ${ }^{13}$ And, those mother rats were declared to suffer gestasional DM since the GDM condition occurred after the rats were declared to be pregnant. DM induction was carried out in this study by injecting streptozotocin in pregnancy day 5 figure 3 .

Enamel thickness was measured using ImageJ software. The measurement of thickness of enamel can be seen in figure 4 .

Figure 4 illustrated that the mean of enamel thickness in the DM group was smaller than in the control group. The results of Mann-Whitney test even showed that there was a significant difference in the enamel thickness between the treatment group and the control group with a p value 0.026 (p $<0.05$ ) It means that DM condition had a significant effect on enamel thickness.

\section{Discussion}

The increased blood glucose levels experienced by the rats may be due to pancreatic beta cell secretions. Streptozotocin is diabetogenic since it can selectively cause necrosis in beta cells that produce insulin. Glucose content in its chemical structure allows STZ to enter the cell through low affinity GLUT2 in the plasma membrane. ${ }^{14}$ The transfer of methyl group of STZ to DNA molecules then cause DNA fragmentation. Protein glycosylation is an additional destructive factor. Poly (ADP-ribose) polymerase (PARP) is stimulated to repair DNA causing decrease in cellular $\mathrm{NAD}^{+}$and ATP deposits, resulting beta cells necrosis. The diabetogenic effect of streptozotocin is also due to its ability to act as a Nitric Oxide (NO) donor. In addition. streptozotocin is able to produce Reactive Oxygen Species (ROS), including hydroxyl and superoxide radicals derived from dismutation of hydrogen peroxide during hypoxanthine metabolism. ${ }^{15}$

The results of this study were actually in line with the results of a previous study which showed a thinner of enamel in the incisors of rat offsprings born to mother rats with alloxan-induced diabetes. ${ }^{4}$ The depletion of the enamel was caused by developmental impediments in early pregnancy when the teeth were in enamel matrix formation phase. ${ }^{16}$ Widening of interstitial spaces in the stellate reticulum inhibit nutrients to secretory ameloblast resulting in decreased secretion of enamel matrix and decreased enamel matrix secretion. The decrease in enamel thickness may also be due to a decrease in glucose in ameloblasts. ${ }^{17}$ Reduced glucose in most cells may cause intracellular metabolic disorder. The metabolite product resulted then can cause morphological changes elsewhere. ${ }^{18}$ 
Another possible causes of decrease of enamel thickness was lack of nutrition. In this study, rat in DM group had lower body weight than those in the control group. This was likely due to impaired carbohydrates, fats and proteins metabolism in the DM group. Fulfillment of nutrition in pregnant mother actually has specific and global effects on the formation of children teeth. Pregnant mother, as a result, must increase their weight to meet the the needs of the fetus and placenta. Maternal weight accompanied by a low protein reserve will increases the risk of enamel hypoplasia in primary or permanent teeth. ${ }^{19}$

Lack of nutrition in DM rats can also cause malnutrition in rat offsprings. Unfortunately, the weight of rat offsprings was not measured in this study. But, the DM group visually had a smaller body size than the control group. Vascular abnormalities that occur in Gestational DM cause protein energy malnutrition leading to slow fetal growth. ${ }^{20}$ Malnutrition can also increase the risk of enamel hypoplasia. ${ }^{21,22}$ Protein is needed in the formation of enamel. Protein energy malnutrition lead to ameloblast disfunction so that enamel matrix secretion decreases. ${ }^{23}$

\section{Conclusion}

It can be concluded that enamel thickness on Gestational Diabetes Mellitus offspring was decrease compared to control. Need to conduct further research on the mechanism of how the enamel thickness decrease.

\section{Acknowledgment}

The authors are grateful to Faculty of Dentistry Universitas Lambung Mangkurat, Indonesia in collaboration with Faculty of Dentistry Universitas Gadjah Mada, Indonesia for supporting this study and also to the Ministry of Research, Technology and Higher Education of the Republic of Indonesia for funding this Research.

\section{Conflict of Interest}

The authors report no conflict of interest.

\section{References}

1. Bath-Balogh M, Fehrenbach MJ. Tooth Development and Eruption. In Dental Embriology, Histology, and Anatomy. 2nd ed. St. Louis: Elseviers Saunders; 2006. p. 61-91.

2. Hovorakova M, Lesot H, Peterka M, Peterkova R. Early development of the human dentition revisited. J Anat 2018;233: 135-145.
3. Aminabadi NA, Oskouei SG, Pouralibaba F, et al. Enamel defects of human primary dentition as virtual memory of early developmental events. J Dent Res Dent Clin Dent Prospect 2009;3: 110-116.

4. Silva-Sousa YTC, Peres LC, Foss MC. Enamel Hypoplasia in a Litter of Rats with Alloxan-Induced Diabetes Mellitus. Braz Dent J 2003;14: 87-93.

5. American Diabetes Association. Diagnosis and Classification of Diabetes Mellitus. Diabetes Care 2005;28(Suppl 1): 37- 42.

6. American Diabetes Association. Gestational diabetes mellitus. Diabetes Care 2003;26(suppl 1): 103-105.

7. Buchanan TA, Xiang AH. Gestational Diabetes Mellitus. J Clin Invest 2005;115: 485-491.

8. Hong L, Levy SM, Warren JJ, Brofffitt B. Association between enamel hypoplasia and dental caries in primary second molars: a cohort study. Caries Res 2009;43: 345-353.

9. Oliveira AF, Chaves AM, Rosenblatt A. The influence of enamel defects on the development of early childhood finding in a population with low socioeconomic status: A longitudinal study. Caries Res 2006;40: 296-302.

10. Hong L, Levy SL, Warren JJ, et al. Association between enamel hypoplasia and dental searches in primary second molars: A cohort study. Caries Res 2009;43: 345-353.

11. Dewi N, Syaify A, Wahyudi IA. Effect of gestational diabetes mellitus on the expression of amelogenin in rat offspring tooth germ. Dent J 2013;46: 83-87.

12. Paronis E, Samara A, Polyzos A, et al. Maternal weight as an alternative determinant of the gestational day of Wistar rats housed in individually-ventilated cages. Lab Anim 2015;49: 188-195.

13. Sharma VK. Streptozotocin: An experimental tool in diabetes and alzheimer's disease (a review). Int J Pharma Res Dev 2010;2: 1-7.

14. Lenzen S. The Mechanisms of alloxan- and streptozotocininduced diabetes. Diabetol 2008;51: 216-226.

15. Soewondo W, Effendi SH. The incidence of enamel hypoplasia and hypocalcification in low birth weight children according to teeth type. J Dent 2012;2: 200-205.

16. Silva-Sousa YTC, Peres LC, Foss MC. Are there structural alterations in the enamel organ of offspring in rats with alloxan-induced diabetes mellitus?. Braz Dent J 2003;14: 162-167.

17. Abbassy MA, Watari I, Bakry AS, et al. Diabetes detrimental effects on enamel and dentine formation. J Dent 2015;43: 589-595.

18. Sheetal A, Hiremath VK, Patil Ag, et al. Manutrition and its oral outcome. J Clin Diagnostic Res 2013;7: 178-180.

19. Nold JL, Georgieff MK. Infants of diabetic mother. Pediatr Clin N Am 2004;51: 619-637.

20. Katge F, Poojari M, Sajnani A. Malnutrition and its effects on oral tissues and dentition. Indian J Dent Edu 2011;4: 45-49.

21. Agarwall S, Narula S, Faridi MMA, et al. Deciduous dentition and enamel defects. Indian Pediatr 2003;40: 124-129.

22. Haggag T, Mahmoud EF. Enamel surface alteration in molars of young rats of diabetic mother. Egyptian Dental Journal 2018;64: 2381-2389.

23. Witzel C, Kierdorf U, Dobney K, et al. Reconstructing impairment of secretory ameloblast function in porcine teeth by analysis of morphological alterations in dental enamel. J Anat 2006;209: 93-110.

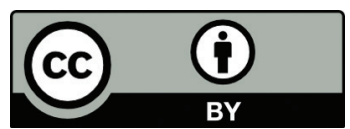

This work is licensed under a Creative Commons Attribution 